\title{
Underwater Image Correction Using Bilateral Filter
}

\author{
Nursanti Abdurrachman ${ }^{1}$, Moh. Jamil ${ }^{2}$ \\ Dept. of Informatics Engineering \\ Universitas Khairun \\ Ternate, Indonesia \\ ${ }^{1}$ nursanti.abdurrachman@gmail.com,.2jamil@unkhair.ac.id
}

\author{
Iis Hamsir Ayub Wahab ${ }^{3}$ \\ Dept. of Electrical Engineering \\ Universitas Khairun \\ Ternate-Indonesia \\ 33hamsir@unkhair.ac.id
}

\begin{abstract}
Bilateral filter is one method of image quality improvement that is nonlinear and adaptive. Bilateral filters combine geometric proximity and colour intensity similarity in mathematical calculations. This method has advantages in improving image quality compared to previous methods. Bilateral filters are used with 3 spatial coefficients and 3 photometric coefficients to process underwater images. The parametersisat used are PSNR and MSE. The results of image processing with bilateral filters are compared with the results of several studies using the trilateral filter. The results show that some studies produce low PSNR values for trilateral and high PSNR values for bilateral filters. From the calculation of Mean Square Error (MSE) and Peak Signal to Noise Ratio (PSNR) results showed that the value of $\sigma s=0.5$ and the value $\sigma R=0.05$ is the best coefficient obtained in the filtering process using a bilateral filter.
\end{abstract}

\section{Keywords-underwater; image; bilateral filter}

\section{INTRODUCTION}

Recently, the development of vision technology becomes very fast. These advances also deliver improvements from objects captured by the image sensor. These are due to the need for the importance of image quality captured in terms of visualization. However, sometimes image quality has decreased or deteriorated due to noise.

Frequent noise is caused by inferiority in the image recording device. In addition, a decrease in image quality can also be caused by interference throughout information transmission, or poor environmental conditions [1] such as underwater images. Absorption of sunlight by water and the exposure of sunlight by small particles in the water environment has become an obstacle to the association's degree to study underwater images with a camera. These can be due to the limited visibility of the camera in exposed sea water and the absorption of sunlight so that the image quality is reduced [2]. Therefore, the need for a method that can restore image quality is higher than before.

Efforts to improve image quality have been widely carried out by researchers, ranging from black and white images to colour images with various filter methods. The method that has been produced from these various studies to improve image quality has also experienced a lot of progress. One method of improving image quality is a bilateral filter. This method is assumed to provide better repairing results than the previous filter method because of its effectiveness in filtering noise while maintaining the edge of the image [3]. Therefore, this study aims to prove this assumption.

\section{BILATERAL FILTER}

Bilateral filters are one method of image quality improvement that is nonlinear and adaptive. Bilateral filters are called nonlinear because the functions used in this method are not linear functions, whereas filters are adaptive because this method does not depend on the type of noise that exists. Bilateral filters combine geometric distances and colour intensity similarities in mathematical calculations. This causes the bilateral filter to have an advantage in improving image quality compared to the previous method [4]. Therefore, this study discusses the improvement of underwater image quality that is damaged by noise interference by using a bilateral filter method.

Mathematically, the bilateral filter shows the weighted average of the pixels of the corrected image $\mathrm{Y}$ given to recover image $X$ as shown in equation (1).

$$
\dot{\mathrm{X}}[k]=\frac{\sum_{n=-N}^{N} W[k, n] Y[k-n]}{\sum_{n=-N}^{N} W[k, n]}
$$

Equation (1) is a normal weighted average of the sample $[2 \mathrm{~N}+1]$ round the kth sample. the weight of $\mathrm{W}[\mathrm{k}, \mathrm{n}]$ is calculated based on neighbouring factors. For a central $\mathrm{X}[\mathrm{k}]$ sample, the weight of $\mathrm{W}[\mathrm{k}, \mathrm{n}]$ is calculated by multiplying the following two factors:

$$
\begin{aligned}
& W_{S}[k, n]=e^{-\frac{d^{2}[k],[k-n]}{2 \sigma_{S}^{2}}} \\
& W_{S}[k, n]=e^{-\frac{n^{2}}{2 \sigma_{S}^{2}}} \\
& W_{R}[k, n]=e^{-\frac{d^{2}\{Y[k], Y[k-n]\}}{2 \sigma_{R}^{2}}} \\
& W_{R}[k, n]=e^{-\frac{\{Y[k], Y[k-n]\}^{2}}{2 \sigma_{R}^{2}}}
\end{aligned}
$$



factors:

The final weight is obtained by multiplying these two

$$
M S E=\frac{1}{M N} \sum_{m=1}^{m}\left(\sum_{n=1}^{n} f\left(\frac{m}{n}\right)-f\left(\frac{m}{n}\right)\right)
$$

The weight of the bilateral filter is temporal (spatial) and radiometric weight. The temporal weight serves to measure the geometric distance between the sample centre [k] and [k-n] of the sample, where the Euclidean metric is used to measure the distance. Thus, the distance of the closest sample will affect the final result compared to a further sample.

The radiometric weight is to measure the radiometric distance between the sample values of the center $\mathrm{Y}[\mathrm{k}]$ and the sample $[\mathrm{k}-\mathrm{n}]$. As with temporal weights, Euclidean metrics are used to measure distances. Thus, samples with the closest distance tend to affect the final yield greater than the distance. In determining the distance value of these two weights, we can use other metrics such as Gaussian functions, symmetrical and decay functions and others.

In bilateral filters, the kernel is applied to the input signal in the k-th sample. This Kernel has the following characteristics as : 1) The number of Kernel coefficients is 1 due to normalization; 2) The center value of the Kernel is the largest. The size depends on the others because of normalization; 3) The kernel can take any form so this can be the basis for better performance.

Bilateral filters are controlled by three parameters, namely: 1) $\mathrm{N}$ which determines filter support where larger values will provide stronger smoothing; 2) Parameters $\sigma s$ and $\sigma \mathrm{R}$. This parameter functions to control the decay of two weight factors. For very large (to infinity) values, this becomes a simple uniform form of non-adaptive filtering, which is commonly known to reduce the edge of the image boundary. Whereas if using a value that is too small it will reduce the smoothing effect [1].

To find out the success of bilateral filters in improving their image and effect on the original image, it was evaluated by measuring Peak Signal to Noise Ratio (PSNR). PSNR is the ratio between possible maximum power and destructive noise that affects image representation. The signal is the original data/image and noise is the error that occurs. PSNR is expressed as a decibel scale and is usually used as a measure of the quality of image reconstruction. The high value of the PSNR shows high image quality [3]. The PSNR equation is as follows [4].

$$
W[k, n]=W_{S}[k, n] . W_{R}[k, n]
$$

where MSE is

$$
\begin{gathered}
P N S R=10 \cdot \log \left(\frac{225^{2}}{M S E}\right) \\
P N S R=20 \cdot \log (225)-10 \cdot \log (M S E)
\end{gathered}
$$

\section{RESULTS}

This research was carried out on underwater images with depths of 1, 2, 3, 4 and 5 meters. The method used for image improvement is a bilateral filter. Bilateral filtering process will be carried out using 3 spatial coefficients of $0.5,3$ and 9,3 photometric coefficients of $0.05,0.5$ and 5 .

Tests are carried out using bilateral filters using 3 spatial coefficients and 3 photometric coefficients have various image results and MSE values and PSNR values. In table, 1 and 2 will be shown the effect of spatial coefficients and photometric coefficients on the image.

TABle I. The Result OF Bilateral Filter

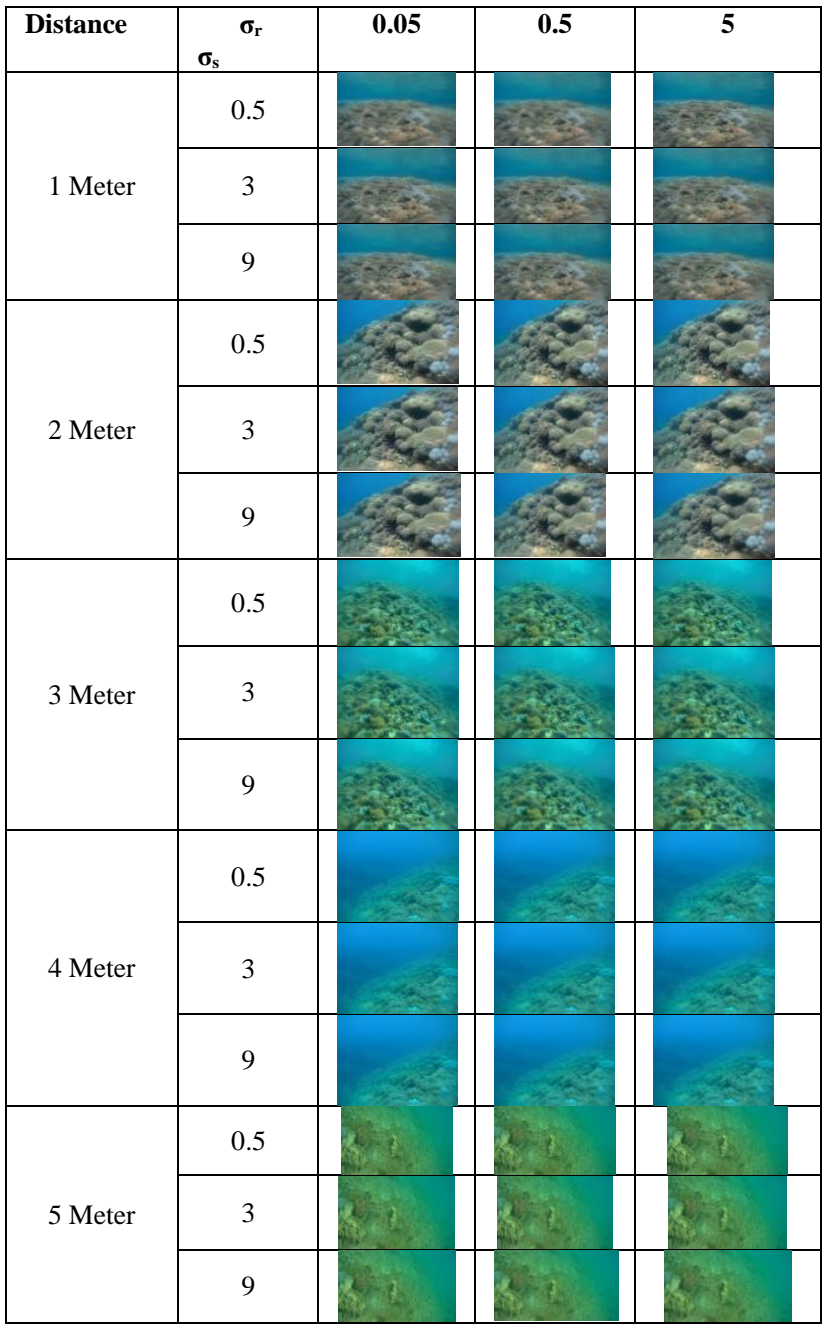

Table 1. The result of bilateral filter for underwater panorama imagery. Figure 2, shows a graph of bilateral filter PSNR results.

The graph shows that sigma_s $=0.5$ and sigma_ $r=0.05$ obtained the highest PSNR results with an average value of 242,277 . Based on the test results, it can be seen that at $\sigma \mathrm{s}=$ 0.5 the bilateral filter method does not have much effect on the image even with different $\sigma \mathrm{R}$. This is due to the lack of strength $\sigma \mathrm{s}$ in spatial weighting. But at $\sigma \mathrm{s}=9$, the weight is 
spatially large so that it affects the firmness of the line or edge of the object in the image. This causes the image to become blurry, as evidenced by the low PSNR value $\sigma \mathrm{s}=9$ compared to the other PSNR values $\sigma \mathrm{s}$ and in accordance with the research conducted by [5]. Then for $\sigma \mathrm{s}=3$, the spatial weighting also affects the firmness of the lines or edges of objects in the image if $\sigma \mathrm{R}=0.5$ and $\sigma \mathrm{R}=5$ so that the image becomes unclear (blur). ) This is proved by the low PSNR value $\sigma \mathrm{R}=0.5$ and $\sigma \mathrm{R}=5$ compared to the PSNR value $\sigma \mathrm{R}=$ 0.05 .

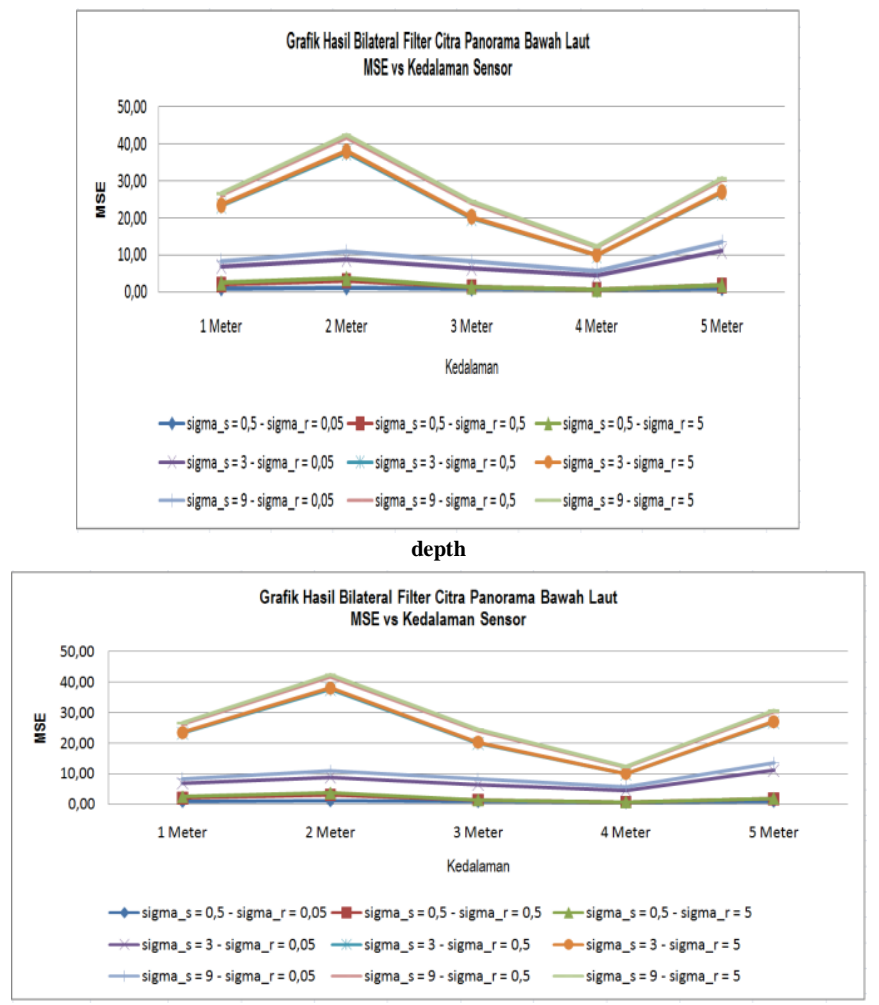

Fig. 1. MSE Results of Bilateral Filters for Underwater Panorama Imagery

While at $\sigma \mathrm{R}=0.05$, it does not give a big impact on photometric weighting. This is evidenced by the low value of MSE. The best filtering results on the bilateral filter method are $\sigma \mathrm{s}=0.5$ and $\sigma \mathrm{R}=0.05$. This can be seen from the high $\mathrm{PSNR}=50.23$ and MSE $=0.62$ in the colour image and the PSNR $=55.89$ and MSE $=0.17$ in the image with the grey level.

\section{CONCLUSION}

In this study, we used bilateral filters with 3 spatial coefficients and 3 photometric coefficients to process underwater images. Then the parameters used are PSNR and MSE. The results of image processing with bilateral filters are compared with the results of several studies using the trilateral filter. The results show that some studies produce low PSNR values for trilateral and high PSNR values for bilateral filters.

\section{REFERENCES}

[1] M. Elad, "On the Origin of the Bilateral Filter and Ways to Improve It," vol. 11, no. 10, pp. 1141-1151, 2002.

[2] S. Swathi and V. Magudeeswaran, "Analysis and Comparison of Different Image Denoising Techniques- Review," vol. 2, pp. 115-124, 2014.

[3] P. Kaushik and Y. Sharma, "Comparison Of Different Image Enhancement Techniques Based Upon Psnr \& Mse," vol. 7, no. 11, 2012.

[4] N. Thakur and S. Devi, "A New Method for Color Image Quality Assessment," vol. 15, no. 2, pp. 10-17, 2011.

[5] L. Liu, C. L. P. Chen, Y. Zhou, and X. You, "A new weighted mean filter with a two-phase detector for removing impulse noise," Inf. Sci. (Ny)., vol. 315, pp. 1-16, 2015 